\title{
Amplitude de movimento de quadril e joelho associada ao índice de massa corporal em
}

\author{
idosos \\ Amplitude of hip and knee movement associated with body mass index in elderly \\ Rango del movimiento de cadera y rodilla asociado al indice de masa corporal en ancianos
}

Recebido: 13/02/2021 | Revisado: 20/02/2021 | Aceito: 04/03/2021 | Publicado: 11/03/2021

\author{
Antonio Vinícius Barros da Silva \\ ORCID: https://orcid.org/0000-0002-0137-9409 \\ Centro Universitário de Ciências e Tecnologia do Maranhão, Brasil \\ E-mail: barrosvinicius719@gmail.com \\ Jeniele de Sousa Silva \\ ORCID: https://orcid.org/0000-0002-2692-5015 \\ Centro Universitário de Ciências e Tecnologia do Maranhão, Brasil \\ E-mail:2017jenisilva@gmail.com \\ Kelly Pereira Rodrigues dos Santos \\ ORCID: https://orcid.org/0000-0002-3483-2425 \\ Centro Universitário de Ciências e Tecnologia do Maranhão, Brasil \\ E-mail: kelly.santos@unifacema.edu.br \\ Cirley Pinheiro Ferreira \\ ORCID: https://orcid.org/0000-0003-3632-7358 \\ Centro Universitário de Ciências e Tecnologia do Maranhão, Brasil \\ E-mail: cirley.prof@gmail.com
}

\begin{abstract}
Resumo
Objetivo: Verificar a amplitude de movimento de quadril e joelho e a associação com IMC em idosos. Metodologia: Trata-se de uma pesquisa bibliográfica do tipo revisão bibliográfica, nas bases de dados Google Acadêmico, SciELO, LILACS e PUBMED no período de 2010 a 2020, onde se estruturou a estratégia PICO por meio da associação dos descritores. Resultados: Foram incluídos oito estudos, havendo uma prevalência de publicações no ano de 2013, dos quais $6(75 \%)$ foram publicações no idioma português, e $2(25 \%)$ na língua inglesa. Os resultados indicam que a prática de exercício físico envolvendo força, mobilidade e flexibilidade melhoram a aptidão física, composição corporal e amplitude de movimento (ADM) nos idosos, apesar da idade e do índice de massa corporal (IMC) influenciarem diretamente na ADM do quadril. Conclusão: Pode-se concluir que o processo de envelhecimento se apresenta de maneira individualizada em cada indivíduo podendo sofrer influência do IMC assim como outras variáveis (idade, ambiente, nível de atividade física), e podem interferir nos parâmetros de ADM dos idosos, em especial na articulação do quadril e a atividade física tem sido uma importante aliada na aquisição ou manutenção desta capacidade física.
\end{abstract}

Palavras-chave: Idoso; Amplitude de movimento articular; Índice de massa corporal; Flexibilidade.

\begin{abstract}
Objective: To check the range of hip and knee motion and the association with BMI in the elderly. Methodology: This is a bibliographic review bibliographic research, in the Google Academic databases, SciELO, LILACS and PUBMED in the period 2010 to 2020, where the PICO strategy was structured through the association of the descriptors. Results: Eight studies were included, with a prevalence of publications in the year 2013, of which $6(75 \%)$ were publications in the Portuguese language, and 2 (25\%) in the English language. The results indicate that the practice of physical exercise involving strength, mobility and flexibility improve physical fitness, body composition and range of motion (ROM) in the elderly, despite the age and body mass index (BMI) influence directly in the range of hip motion. Conclusion: It can be concluded that the aging process is individually located in each individual and may influence the BMI as well as other variables (age, environment, level of physical activity), and may interfere with the elderly parameters of the elderly, special in hip joint and physical activity has been an important ally in the acquisition or maintenance of this physical capacity.
\end{abstract}

Keywords: Aged; Range of motion; Body mass index; Pliability.

\section{Resumen}

Objetivo: Verificar el rango del movimiento de cadera y rodilla y la asociación con IMC en los ancianos. Metodología: Esta es una investigación bibliográfica de revisión bibliográfica, en las bases de datos académicas de Google, SciELO, LILACS y PUBMED en el período 2010 a 2020, donde la estrategia PICO se estructuró mediante la asociación de 
descriptores. Resultados: se incluyeron ocho estudios, con una prevalencia de publicaciones en el año 2013, de las cuales $6(75 \%)$ fueron publicaciones en el idioma portugués y $2(25 \%)$ en el idioma inglés. Los resultados indican que la práctica del ejercicio físico que involucra fuerza, movilidad y flexibilidad mejore la aptitud física, la composición corporal y el rango de movimiento en los ancianos, a pesar de la edad y el índice de masa corporal influyen directamente en el rango del movimiento de cadera. Conclusión: se puede concluir que el proceso de envejecimiento se encuentra individualmente en cada individuo y puede influir en el índice de masa corporal, así como otras variables (edad, medio ambiente, nivel de actividad física), y pueden interferir con los parámetros del rango del movimiento de los ancianos, especialmente en la articulación de la cadera y la actividad física ha sido un aliado importante en la adquisición o mantenimiento de esta capacidad física.

Palabras clave: Anciano; Rango del movimiento articular; Índice de masa corporal; Docilidad.

\section{Introdução}

Envelhecer pode ser definido como um processo dinâmico e progressivo, entretanto as alterações decorrentes deste processo são mais visíveis com o chegar da terceira idade. Segundo a Organização Mundial de Saúde (OMS) até 2050 a população mundial contará com um número expressivo de indivíduos com idade superior a 60 anos, com maior concentração nos países desenvolvidos (Camões et al., 2016).

O envelhecimento caracteriza-se pela convergência de várias alterações corporais como a diminuição da densidade óssea, flexibilidade, massa muscular, habilidades funcionais e/ou aumento da adiposidade corporal, além da redução no desempenho cognitivo, podendo provocar complicações e a incidência de doenças crônicas (Oliveira et al., 2019).

A inatividade física torna-se um risco que provoca vários efeitos negativos ao organismo da pessoa idosa, prejudicando assim a saúde e consequentemente sua qualidade de vida. Durante o processo de envelhecimento ocorre também a diminuição da mobilidade, limitando assim os seus movimentos e a amplitude dos mesmos, que são indispensáveis para a realização de suas atividades cotidianas (Santos et al., 2015 \& Vaz, 2014).

A prática regular de exercício físico desenvolve uma melhora na aptidão física e cognitiva dos idosos, podendo prevenir e/ou reverter possíveis complicações adquiridas no processo de envelhecimento, todavia, é expressivo o número de idosos sedentários (Oliveira et al., 2019).

A avaliação da amplitude de movimento (ADM) tem sido bastante útil na quantificação do déficit musculoesquelético, além de servir como base para avaliar a eficácia das intervenções terapêuticas. Através da ADM é possível mensurar a capacidade articular dos membros, que são indispensáveis na realização das atividades diárias dos idosos, assim como na realização de atividades físicas (Lima et al., 2012). Profissionais da saúde fazem o uso de diversos instrumentos como goniômetros, flexímetro, inclinômetro, orthoranger e estimação visual para mensurar e avaliar a (ADM) amplitude de movimento articular (Prado, 2018).

Os altos índices de obesidade existentes em idosos, descrevem que a nutrição é um aspecto importante neste contexto pela modulação das mudanças fisiológicas relacionadas com a idade e no desenvolvimento de doenças crônicas não transmissíveis, como doenças cardiovasculares, diabetes, obesidade, osteoporose e alguns tipos de câncer (Vellozo \& Fisberg, 2010).

A atividade física supervisionada é de suma importância nesse processo para que estes idosos consigam manter uma boa qualidade de vida e possam desenvolver suas atividades com mais independência, pois com exercícios regulares poderão fortalecer seus sistemas corporais, e tornarem-se menos susceptíveis a lesões.

Tendo em vista a relevância do estudo da amplitude de movimento para o idoso na prevenção ou redução de quedas, o presente estudo tem como objetivo verificar a ADM de quadril e joelho e a associação com índice de massa corporal em idosos, revestindo-se de importância o desenvolvimento de estudos para garantir a saúde e manutenção da qualidade de vida desse público.

\section{Metodologia}

Trata-se de uma pesquisa de revisão bibliográfica, de caráter quantitativo. Método que segundo segundo Pereira et al., 
(2018) caracteriza-se pela utilização de técnicas matemáticas e métodos estatísticos que podem proporcionar previsões em relação ao objeto de estudo da pesquisa. Determinou-se a construção da estratégia PICO, que representa o acrômio para (P) paciente ou população, (I) intervenção, (C) comparação ou controle, $(\mathrm{O})$ desfecho ou resultado, na qual foi utilizada para a elaboração da seguinte questão norteadora desta revisão de literatura integrativa: "Qual a possível associação da amplitude de movimento de quadril e joelho com índice de massa corporal em idosos?".

Para a busca de materiais bibliográficos foram utilizados descritores no idioma português e inglês cadastrados no Descritores em Ciências da Saúde, sendo utilizados na pesquisa do material bibliográfico nas seguintes bases de dados: Google Acadêmico, SciELO (Scientific Electronic Library Online), LILACS (Literatura Latino-Americana e do Caribe em Ciências da Saúde) e PUBMED (National Library of Medicine).

Aplicando na pesquisa o filtro limitando-se a inclusão de estudos com textos completos, idioma português e inglês, publicados no período de 2010 a 2020 foram analisados e selecionados 2 (dois) estudos no Google Acadêmico, na base de dados SCIELO obteve-se 18 (dezoito) estudos onde apenas 2 (dois) estudos foram condizentes com a questão norteadora desta pesquisa, na base de dados LILACS foram selecionados apenas 1 (um) estudo, e na plataforma PUBMED foram selecionados 3 (três) estudos, resultando em 8 artigos publicados entre 2012 e 2018, estes que foram utilizados na discussão da pesquisa.

$\mathrm{Na}$ etapa de análise e interpretação dos resultados foram analisados os materiais bibliográficos e criadas categorias analíticas que proporcionaram a ordenação e a síntese de cada estudo. Essa categorização foi efetivada de forma descritiva, indicando os dados mais importantes para o estudo.

Tabela 1. Elementos da estratégia PICO, descritores e palavras-chave.

\begin{tabular}{|c|c|c|c|}
\hline & Elementos & Decs & Palavras-Chave \\
\hline $\mathbf{P}$ & Idoso & $\begin{array}{l}\text { "Idoso", } \\
\text { "Elderly" }\end{array}$ & $\begin{array}{l}\text { "Idoso", } \\
\text { "Elderly" }\end{array}$ \\
\hline I & Amplitude de movimento & $\begin{array}{l}\text { "Amplitude de Movimento" } \\
\text { "Range of Motion" }\end{array}$ & $\begin{array}{l}\text { "Amplitude de Movimento" } \\
\text { "Range of Motion" }\end{array}$ \\
\hline $\mathbf{C}$ & $\begin{array}{l}\text { Amplitude de movimento } \\
\text { Índice de massa corporal }\end{array}$ & $\begin{array}{l}\text { "Amplitude de Movimento" } \\
\text { "Range of Motion" } \\
\text { "Índice de massa corporal" } \\
\text { "Body Mass Index" }\end{array}$ & $\begin{array}{l}\text { "Amplitude de Movimento" } \\
\text { "Range of Motion" } \\
\text { "Índice de Massa Corporal" } \\
\text { "Body Mass Index" }\end{array}$ \\
\hline $\mathbf{O}$ & Diminuição flexibilidade & $\begin{array}{l}\text { "Flexibilidade" } \\
\text { "Flexibility" }\end{array}$ & $\begin{array}{l}\text { "Flexibilidade" } \\
\text { "Flexibility" }\end{array}$ \\
\hline
\end{tabular}

Fonte: Dados da pesquisa (2020).

Na Tabela 1 estão representados os elementos utilizados na construção da estratégia PICO, e seus respectivos descritores e palavras-chave no idioma inglês e português. 


\section{Resultados}

Tabela 2. Distribuição da amostra referente ao ano de publicação.

\begin{tabular}{c|c|c}
\hline PERÍODO DE PUBLICAÇÃO & NÚMERO ABSOLUTO & $(\%)$ \\
\hline 2012 & 02 & 25 \\
\hline 2013 & 03 & 37,5 \\
\hline 2015 & 01 & 12,5 \\
\hline 2016 & 01 & 12,5 \\
\hline 2018 & 01 & 100 \\
\hline TOTAL & 08 & \\
\hline
\end{tabular}

Fonte: Dados da pesquisa (2020).

Em relação ao período de publicação dos artigos selecionados na discussão da pesquisa, houve uma prevalência de publicações do ano de 2013, contando com três artigos datados neste período conforme representa a Tabela 2.

Tabela 3. Demonstrativo dos artigos utilizados na elaboração dos resultados e discussão da pesquisa.

\begin{tabular}{|c|c|c|c|}
\hline AUTORES/ANO & $\begin{array}{c}\text { TIPO DE } \\
\text { ESTUDO/AMOSTR } \\
\text { A }\end{array}$ & OBJETIVO & CONSIDERAÇÕES FINAIS \\
\hline $\begin{array}{l}\text { Kouyoumdjian } \\
\text {; Coulomb; } \\
\text { Sanchez; } \\
\text { Asencio (2012). }\end{array}$ & $\begin{array}{c}\text { Prospectivo } \\
\text { diagnóstico: nível } \\
\text { III/120 participantes. }\end{array}$ & $\begin{array}{c}\text { Atualizar os dados sobre a } \\
\text { ADM de rotação passiva do } \\
\text { quadril em adultos usando } \\
\text { um método mais confiável de } \\
\text { medição fotográfica }\end{array}$ & $\begin{array}{l}\text { O estudo apresentou evidências que a idade, } \\
\text { IMC e sexo são fatores determinantes que } \\
\text { influenciam diretamente na amplitude de } \\
\text { movimento do quadril. }\end{array}$ \\
\hline $\begin{array}{l}\text { González; Sánchez; } \\
\text { Santos (2012). }\end{array}$ & $\begin{array}{l}\text { Projeto de } \\
\text { intervenção pré- } \\
\text { treinamento e pós- } \\
\text { treinamento/54 } \\
\text { participantes. }\end{array}$ & $\begin{array}{c}\text { Determinar a influência de } 2 \\
\text { métodos de treinamento } \\
\text { elástico (facilitação } \\
\text { neuromuscular passiva e } \\
\text { proprioceptiva [PNF]) na } \\
\text { amplitude de movimento } \\
\text { (ADM) em pessoas idosas } \\
\text { entre } 60 \text { e } 70 \text { anos, durante } \\
\text { um período de } 13 \text { semanas }\end{array}$ & $\begin{array}{l}\text { Foi verificado que a capacidade dos idosos } \\
\text { ativos melhorarem a ADM em resposta às } \\
\text { técnicas de alongamento é semelhante para } \\
\text { as técnicas passiva e PNF. }\end{array}$ \\
\hline $\begin{array}{c}\text { Fidelis; } \\
\text { Patrizzi; } \\
\text { Walsh, (2013). }\end{array}$ & $\begin{array}{l}\text { Estudo descritivo, } \\
\text { transversal, de caráter } \\
\text { exploratório e } \\
\text { metodologia } \\
\text { quantitativa/74 } \\
\text { idosos. }\end{array}$ & $\begin{array}{l}\text { Avaliar os resultados da } \\
\text { prática de exercícios físicos } \\
\text { sobre a força muscular } \\
\text { manual, flexibilidade e } \\
\text { mobilidade funcional em } \\
\text { idosos usuários de unidades } \\
\text { de saúde da cidade de } \\
\text { Uberaba-MG }\end{array}$ & $\begin{array}{l}\text { Os dados da pesquisa apontaram a } \\
\text { importância de melhorar a qualidade da } \\
\text { prática de exercícios físicos oferecidos } \\
\text { pelas unidades básicas de saúde, } \\
\text { trabalhando principalmente a flexibilidade, } \\
\text { mobilidade e força muscular. }\end{array}$ \\
\hline Silva et al., (2013). & $\begin{array}{l}\text { Estudo transversal, } \\
\text { domiciliar/420 } \\
\text { idosos. }\end{array}$ & $\begin{array}{l}\text { Verificar a correlação da } \\
\text { força de preensão manual e } \\
\text { flexibilidade com idade e } \\
\text { variáveis antropométricas em } \\
\text { idosos. }\end{array}$ & $\begin{array}{l}\text { O estudo sugere que as limitações em } \\
\text { relação a flexibilidade podem estar } \\
\text { associadas à idade, assim como as variáveis } \\
\text { antropométricas e composição corporal do } \\
\text { idoso. }\end{array}$ \\
\hline
\end{tabular}




\begin{tabular}{|c|c|c|c|}
\hline Vila et al., (2013). & $\begin{array}{l}\text { Estudo observacional } \\
\text { analítico do tipo } \\
\text { transversal/25 idosas. }\end{array}$ & $\begin{array}{c}\text { Analisar a aptidão física } \\
\text { funcional e a atenção, entre } \\
\text { dois grupos de idosas } \\
\text { praticantes de exercício físico } \\
\text { e sedentárias. }\end{array}$ & $\begin{array}{l}\text { Os achados da pesquisa apontam que as } \\
\text { idosas ativas apresentaram uma } \\
\text { superioridade nos níveis de flexibilidade, } \\
\text { mobilidade e capacidade aeróbica em } \\
\text { relação as idosas não ativas. }\end{array}$ \\
\hline $\begin{array}{l}\text { Zambon et al., } \\
\qquad(2015) .\end{array}$ & $\begin{array}{c}\text { Estudo é } \\
\text { observacional e } \\
\text { transversal/60 idosas. }\end{array}$ & $\begin{array}{l}\text { Comparar a flexibilidade } \\
\text { de mulheres idosas } \\
\text { praticantes hidroginástica, } \\
\text { treinamento combinado e não } \\
\text { ativas. }\end{array}$ & $\begin{array}{l}\text { Os resultados do estudo indicam que a } \\
\text { prática da hidroginástica e treinamento } \\
\text { combinado proporcionam uma melhora dos } \\
\text { níveis de flexão e extensão do quadril das } \\
\text { idosas. }\end{array}$ \\
\hline Brito et al., (2016). & $\begin{array}{l}\text { Pesquisa diagnóstica, } \\
\text { descritiva, } \\
\text { exploratória com } 22 \\
\text { alunas das aulas de } \\
\text { alongamentos. }\end{array}$ & $\begin{array}{c}\text { Avaliar o nível de } \\
\text { flexibilidade, o índice de } \\
\text { massa corporal (IMC) e a } \\
\text { imagem corporal de um } \\
\text { grupo de idosas praticantes } \\
\text { de alongamento no Centro } \\
\text { Desportivo Municipal } \\
\text { (CDM). }\end{array}$ & $\begin{array}{l}\text { Verificou-se que a prática do exercício } \\
\text { físico é uma ferramenta importância para a } \\
\text { saúde da população idosa, melhorando os } \\
\text { níveis de flexibilidade, proporcionando } \\
\text { indepêndencia em relação a realização das } \\
\text { atividades cotidianas das mesmas. }\end{array}$ \\
\hline $\begin{array}{l}\text { Fonseca et al., } \\
\text { (2018). }\end{array}$ & $\begin{array}{l}\text { Estudo longitudinal } \\
\text { com coleta de dados } \\
\text { em idosos. }\end{array}$ & $\begin{array}{c}\text { Avaliar os efeitos de um } \\
\text { programa de treinamento de } \\
\text { força nos indicadores de } \\
\text { composição corporal e } \\
\text { aptidão física funcional de } \\
\text { idosos ativos praticantes de } \\
\text { musculação. }\end{array}$ & $\begin{array}{l}\text { Os resultados indicaram melhora da aptidão } \\
\text { física funcional e composição corporal dos } \\
\text { idosos após a intervenção, sugerindo que a } \\
\text { prática do treinamento de força é uma } \\
\text { possibilidade que proporciona de maneira } \\
\text { eficiente a melhora destes índices. }\end{array}$ \\
\hline
\end{tabular}

Fonte: Dados da pesquisa (2020).

A Tabela 3 esboça o esquema de distribuição do material bibliográfico utilizado na discussão da pesquisa em relação a (s) autor (es), ano de publicação, tipo de estudo, amostra, objetivo e considerações finais.

Quadro 1. Distribuição dos artigos de acordo com o idioma.

\begin{tabular}{|c|c|c|}
\hline IDIOMA & NÚMERO ABSOLUTO & $\%$ \\
\hline PORTUGUÊS & 6 & 75 \\
\hline INGLÊS & 2 & 25 \\
\hline TOTAL & 8 & 100 \\
\hline
\end{tabular}

Fonte: Dados da pesquisa (2020).

O Quadro 1 representa a distribuição dos estudos em relação ao idioma, havendo prevalência de $75 \%$ de publicações em português.

\section{Discussão}

Em seu estudo Silva et al. (2013), relata que nesta fase da vida ocorrem alterações corporais que interferem na habilidade e autonomia do indivíduo. Também associa o índice de massa corporal com a flexibilidade e amplitude de movimento, ou seja, propõe que indivíduos idosos com sobrepeso ou obesidade apresentam maiores limitações na realização de movimentos.

Corroborando com este estudo Kouyoumdjian; Coulomb, Sanchez e Asencio (2012), também classificam as variáveis 
idade, sexo e índice de massa corporal como fatores determinantes que influenciam diretamente na amplitude de movimento da articulação do quadril. Gonzáles (2012), categoriza a flexibilidade como uma habilidade física que proporciona indepedência à pessoa idosa na realização das atividades cotidianas e ressalta que o envelhecimento reduz a amplitude dos movimentos podendo aumentar o risco de quedas, porém destaca que a prática de exercícios físicos melhora esta capacidade funcional.

Nesta mesma perspectiva Fidelis, Patrizzi e Walsh (2013), apontam que a prática regular de exercícios físicos atua de maneira positiva, proporcionando a manutenção dos níveis de aptidão física. A respeito disso Vila et al. (2013), em estudo comparativo de idosas praticantes de exercícios físicos e sedentárias constatou que as idosas ativas apresentaram melhores resultados em relação aos níveis de flexibilidade das articulações de membros inferiores, mobilidade e resistência aeróbica. Também destaca que a prática de exercícios de flexibilidade além de melhorar a ADM, reduz o acometimento de dores.

Em estudo realizado com 22 mulheres idosas Brito et al. (2016), verificou que as mesmas possuíam níveis de flexibilidade considerados adequados para uma vida saudável devido a prática regular de alongamentos, sugerindo que esta atividade melhora a amplitude articular contribuindo positivamente no processo de envelhecimento dessas idosas.

Já Zambon et al. (2015), em seu estudo comparativo com 60 mulheres idosas praticantes de hidroginástica, treinamento combinado e não praticantes observou que essas atividades físicas melhoram a amplitude articular do quadril, porém não foi encontrada influência significativa da prática desses exercícios físicos em relação ao IMC das participantes.

Em estudo realizado com uma amostra composta por 12 idosos de ambos os sexos, praticantes de musculação Fonseca et al. (2018), evidenciou que a prática periodizada do treinamento de musculação apresentou-se como uma possibilidade eficaz para uma melhora nos índices de aptidão física funcional e composição corporal dos idosos.

\section{Conclusão}

A análise dos estudos define o envelhecimento como um processo dinâmico, progressivo e individual em cada pessoa, podendo sofrer influência de diversos fatores. Entre os principais fatores existem as alterações corporais, com destaque para o declínio das capacidades físicas funcionais de flexibilidade, amplitude de movimento provocando limitações na realização de atividades diárias e aumento da adiposidade corporal, aumentando assim o risco de incidência de doenças crônicas provocando o comprometimento da qualidade de vida do indivíduo.

Também foi verificado que o índice de massa corporal assim como outras variáveis (idade, ambiente, nível de atividade física), apresentaram-se como fatores determinantes que interferem nos parâmetros de amplitude de movimento de idosos, em especial na articulação do quadril e que a atividade física é apontada como uma aliada na aquisição ou manutenção desta capacidade física.

Sobre a temática abordada, sugere-se novas pesquisas que busquem mais evidências da possível associação da amplitude de movimento de joelho e quadril com o índice de massa corporal em idosos, contribuindo para um melhor esclarecimento da problemática, que pouco tem sido relatada na literatura.

\section{Referências}

Brito, E., Prade, L. C., Siqueira, L. Q., Daronco, L. S. E., Leiria, M. P. \& Valente, W. P. (2016). Flexibilidade, imagem corporal e índice de massa corporal de idosas praticantes de alongamento no Centro Desportivo Municipal (CDM) Santa Maria/2015. Rev. Salusvita. 35(4), 477-487. https://pesquisa.bvsalud.org/portal/resource/pt/biblio-836795?lang=fr

Camões, M., Fernandes, F., Silva, B., Rodrigues, T., Costa, N. \& Bezerra, P. (2016) Exercício físico e qualidade de vida em idosos: diferentes contextos sociocomportamentais. Motricidade, 12(10, 96-105. http://www.scielo.mec.pt/scielo.php?script=sci_arttext\&pid=S1646-107X2016000100010

Fidelis, L. T., Patrizzi, L. J. \& Walsh, I. A. P. (2013). Influência da prática de exercícios físicos sobre a flexibilidade, força muscular manual e mobilidade funcional em idosos. Revista Brasileira de Geriatria e Gerontologia, 16(1), 109-116. https://www.scielo.br/scielo.php?pid=S1809- 


\section{1\&script=sci_arttext\&tlng=pt}

Fonseca, A. I. S., Barbossa, T. C., Silva, B. K. R., Ribeiro, H. S., Quaresma, F. R. P. \& Maciel, E. S. (2018). Efeito de um programa de treinamento de força na aptidão física funcional e composição corporal de idosos praticantes de musculação. Revista Brasileira de Prescrição e Fisiologia Do Exercício (RBPFEX), 12(76), 556-563. http://www.rbpfex.com.br/index.php/rbpfex/article/view/1456

González-Ravé, J. M., Sánchez-Gómez, A. \& Santos-García, D. J. (2012). Eficácia de dois programas de treinamento de alongamento diferentes (facilitação neuromuscular passiva vs. proprioceptiva) na amplitude de movimento do ombro e do quadril em idosos. O Journal of Strength \& Conditioning Research, 26(4),.1045-1051. https://journals.lww.com/nsca-jscr/Fulltext/2012/04000/Efficacy_of_Two_Different_Stretch_Training.23.aspx

Kouyoumdjian, P., Coulomb, R., Sanchez, T. \& Asencio, G. (2012). Avaliação clínica da amplitude de movimento da rotação da articulação do quadril em adultos. Ortopedia e Traumatologia: Cirurgia e Pesquisa, 98(1), 17-23, 2012. 10.1016/j.otsr.2011.08.015>

Lima, F. V., Pereira, D. G., Diniz, R. C. R., Santiago, D. C. G., Alves, B. P. \& Chagas, M. H. (2012). Efeito da amplitude de movimento no número máximo de repetições no exercício supino livre. Rev. bras. Educ. Fís. Esporte, 26(4), 571-79 https://www.scielo.br/scielo.php?pid=S1807$55092012000400004 \&$ script=sci_arttext\&tlng=pt

Oliveira, D. V. D., Oliveira, V. B. D., Caruzo, G. A., Ferreira, Á. G., Nascimento Júnior, J. R. A. D., Cunha, P. M. D., \& Cavaglieri, C. R. (2019). O nível de atividade física como um fator interveniente no estado cognitivo de idosos da atenção básica à saúde. Ciência \& Saúde Coletiva, 24, 4163-4170. https://www.scielosp.org/article/csc/2019.v24n11/4163-4170/pt/

Pereira, A. S. et al. (2018). Metodologia da pesquisa científica. http://repositorio.ufsm.br/handle/1/15824.

Prado, R. P. (2018). Mensuração da amplitude de movimento ativa do joelho intra e interavaliador com goniômetro e flexímetro. Fisioterapia Brasil, 8(6), 409-413. http://www.portalatlanticaeditora.com.br/index.php/fisioterapiabrasil/article/view/1813

Santos, R. G., Medeiros, J. C., Schmitt, B. D., Meneguci, J., Santos, D. A. T., Damião, R., Tribess, S. \& Virtuoso Júnior, J. S. (2015). Comportamento Sedentário em Idosos: uma revisão sistemática. Motricidade, 11(3), 171-186. http://www.scielo.mec.pt/scielo.php?pid=S1646107 X2015000300016\&script=sci_arttext\&tlng=en

Silva, N. A, Menezes, T. N., Melo R. L. \& Pedraza, D. F. (2013). Força de preensão manual e flexibilidade e suas relações com variáveis antropométricas em idosos [Handgrip strength and flexibility and their association with anthropometric variables in the elderly]. Rev Assoc Med Bras (1992). 10.1016/j.ramb.2012.10.002. https://www.scielo.br/scielo.php?script=sci_arttext\&pid=S0104-42302013000200011

Vaz, R. C. R. (2014). Envelhecimento e atividade física: influências na qualidade de vida. 2014. Monografia (Curso de Educação Física), Faculdade de Educação Física da Universidade Federal de Goiás, 61p., Goianésia (GO). https://repositorio.bc.ufg.br/bitstream/ri/4608/5/TCCG\%20\% E2\%80\%93\%20Educa\%C3\%A7\%C3\%A30\%20F\%C3\%ADsica\%20-\%20Rosana\%20da\%20Costa\%20Rodrigues.pdf

Vellozo, E. P. \& Fisberg, M. (2010). The impact of food fortification on the prevention of iron deficiency. Rev. Bras. Hematol. Hemoter. vol.32, supl.2, São Paulo. https://www.scielo.br/scielo.php?pid=S1516-84842010005000069\&script=sci_arttext

Vila, C. P., Silva, M. E. M., Simas, J. P. N.; Guimarães, A. C. A. \& Parcias, S. R. (2013). Aptidão física funcional e nível de atenção em idosas praticantes de exercício físico. Revista Brasileira de Geriatria e Gerontologia, 16(2),. 355-364. https://www.scielo.br/scielo.php?pid=S180998232013000200015\&script=sci_arttext

Zambon, T. B., Gonelli, P. R. G., Gonçalves, R. D., Borges, B. L. A., Montebelo, M. I. L. \& Cesar, M. C. (2015). Análise comparativa da flexibilidade de mulheres idosas ativas e não ativas. Revista Acta Fisiátrica, 22(1), 14-18. http://www.revistas.usp.br/actafisiatrica/article/view/103895 